\title{
Curative effect of methotrexate combined with teniposide in the treatment of primary central nervous system lymphoma
}

\author{
YI-XIA WANG ${ }^{1,2}$, YAN HUANG ${ }^{2}$, XIAO-PING XU ${ }^{1}$, BO-BIN CHEN ${ }^{1}$, ZHI-GUANG LIN ${ }^{1}$, \\ YAN MA ${ }^{1}$, TIAN-LING DING ${ }^{1}$ and QIAN WANG ${ }^{1}$ \\ ${ }^{1}$ Department of Hematology, Huashan Hospital Affiliated to Fudan University, Shanghai 200040; \\ ${ }^{2}$ Department of Hematology, The Second People's Hospital of Kashi, Xinjiang 844000, P.R. China
}

Received September 22, 2016; Accepted March 7, 2018

DOI: $10.3892 / \mathrm{ol} .2020 .11328$

\begin{abstract}
The present study aimed to investigate the curative effect of high-dose methotrexate (HD-MTX) combined with teniposide (Vm26) vs. HD-MTX alone in the treatment of primary central nervous system lymphoma (PCNSL), in order to provide data for assisting decisions associated with clinical treatment. Data from 56 patients with PCNSL admitted in Shanghai Huashan Hospital (Shanghai, China) from January 2009 to December 2014 were included into the present study. Clinical data, curative effects and prognosis of patients in these two groups were retrospectively analyzed using SPSS 20 statistical software. In the HD-MTX+Vm26 group, 12 patients $(42.85 \%)$ achieved complete remission (CR) and 10 patients $(35.71 \%)$ achieved partial remission (PR), while in the HD-MTX group 7 patients $(25 \%)$ achieved CR and 11 patients (39.29\%) achieved $\mathrm{PR}(\mathrm{P}=0.158)$. The median progression-free survival (PFS) time was 22 months in the HD-MTX+Vm26 group and 12 months in the HD-MTX group $(\mathrm{P}=0.019)$. The median overall survival time was 57 months in the HD-MTX+Vm26 group, and 28 months in the HD-MTX group $(\mathrm{P}=0.013)$. Compared with HD-MTX alone, the combined treatment of HD-MTX+Vm26 had an improved curative effect in the treatment of PCNSL, effectively controlled tumor progression in patients, prolonged survival time and improved prognosis. Age was an independent prognostic factor in patients with PCNSL. Patients with an age of $\leq 60$ years exhibited longer PFS compared with patients with an age of $>60$ years.
\end{abstract}

\section{Introduction}

Primary central nervous system lymphoma (PCNSL) is a malignant tumor confined to the brain, pia mater, spinal cord

Correspondence to: Dr Xiao-Ping Xu, Department of Hematology, Huashan Hospital Affiliated to Fudan University, 12 Middle Urumqi Road, Shanghai 200040, P.R. China

E-mail: xpxu123doc@163.com

Key words: methotrexate, teniposide, primary central nervous system lymphoma, curative effect, prognosis or eye (1). A study at the Massachusetts General Hospital from 1958 to 1989 showed that a total of $>90 \%$ of patients with PCNSL have diffuse large B cell lymphoma (2), which exhibits high invasion rates and poor prognosis. Poor prognostic factors include age, Karnofsky score, serum lactate dehydrogenase (LDH), cerebrospinal fluid protein, B cell lymphoma 6 protein expression (3) and reduced lymphocytes (4).

PCNSL cells usually present with diffuse infiltration. Therefore, it is difficult to completely remove by surgery; and its recurrence rate is high (5). Previously, whole brain radiotherapy was performed as a first-line therapy; however, treatment with radiotherapy alone confers disease recurrence and neurotoxicity $(6,7)$, which limits its clinical application. High-dose methotrexate (HD-MTX) has an affirmed curative effect, but the complications, such as neurotoxicity, limit its dose $(8,9)$. In addition, the 2-year overall survival (OS) rate of HD-MTX monotherapy is only $61-63 \%(10,11)$, which is not satisfying. At present, the preferred regimen is the combined treatment based on HD-MTX (combined with cytarabine, rituximab and idarubicin) (12-18). However, the combination of HD-MTX based chemotherapy is still under debate. Teniposide (Vm26) has high liposolubility, which makes it is able to pass the blood-brain barrier, and it has a slower elimination rate (19). Fan et al (20) Revealed that MTX+Vm26 did not significantly improve the median progression-free survival of patients, but complete remission (CR) and overall response (OR) were significantly increased. In the present study, the clinical curative effects of HD-MTX+Vm26 vs. Vm26 alone were compared, with the aim of providing a useful reference for clinical practice.

\section{Materials and methods}

Data acquisition. A total of 56 patients with PCNSL who were admitted, but had not been treated, to the Department of Hematology of Huashan Hospital Affiliated to Fudan University (Shanghai, China) from January 2009 to December 2014 were enrolled into the present study. Due to the retrospective nature of the present study, the requirement for ethical approval was waived by the Ethics Committee of Huashan Hospital (Shanghai, China). The cohort of 56 patients comprised 38 males and 18 females. The age of these patients 
ranged between $25-74$ years, with a median age of 54.5 years. A total of 13 patients $(23.21 \%)$ were $\geq 60$ years.

According to the treatment regimen received, patients were divided into two groups: A HD-MTX+Vm26 group and HD-MTX group ( $\mathrm{n}=28$ in each group). A retrospective analysis method was adopted. Inclusion criteria were as follows: i) Patients who were first diagnosed with PCNSL; ii) patients with a negative HIV test result; iii) patients with diagnosis supported by pathological evidences; iv) patients who had completed at least three courses of chemotherapy; v) patients with changes in intracranial lesions that had been followed up by imaging tests (MRI or PET-CT) prior to diagnosis and subsequent to chemotherapy; and vi) patients who were diagnosed based on the 2008 World Health Organization classification of hematopoietic and lymphoid neoplasms (21). Exclusion criteria were as follows: Congenital or acquired immunodeficiency, including patients with previous organ transplantation, concurrent treatment with immunosuppressive drugs, and AIDS-related PCNSL; disease confined to the eye without another localization in the central nervous system (CNS); the presence or history of systemic lymphoma; any prior malignancy with the exception of adequately treated nonmelanoma skin cancer and carcinoma in situ of the cervix uteri; a serious impairment of cardiac, renal, or liver function; pregnancy; any severe uncontrolled infection; prior chemotherapy, with the exception of corticosteroids, for a maximum period of 6 weeks before and after diagnosis or surgery; and Burkitt's lymphomas of low-grade T-cell lymphomas.

Tissue and reagents. 56 cases of excised tissue or biopsy tissue of brain tumor were collected, fixed by $3.7 \%$ neutral formaldehyde at room temperature for 6-12 h, until further processing, routinely dehydrated, embedded by paraffin, sliced into $3-\mu \mathrm{m}$ thick sections, and then subjected to $H \& E$ staining (stained with Gill's hematoxylin for 2-3 min and eosin for 10-20 sec at room temperature) and immunohistochemical staining. In immunohistochemical staining, the tissue was embedded by paraffin, sliced into $4-\mu \mathrm{m}$ thick sections, dewaxed with $100 \%$ xylene and concentration gradients of alcohol (100\% EtOH for 5 min twice, 95\% $\mathrm{EtOH}$ for $4 \mathrm{~min}$ twice, $80 \% \mathrm{EtOH}$ for $4 \mathrm{~min}$ ) according to the standard operation process, and repaired in citrate buffer (pH 6.0; Shanghai Xin Yu Biotech Co., Ltd, Shanghai, China) and boiled for $10 \mathrm{~min}$. After natural cooling, the endogenous peroxidase activity was blocked using $3 \%$ hydrogen peroxide at room temperature for $10 \mathrm{~min}$, and the sections were blocked using 10\% goat serum/PBS at room temperature for $30 \mathrm{~min}$, then and treated with the primary antibodies and incubated at $4^{\circ} \mathrm{C}$ overnight: CD10 (1:50; cat. no. ab951), CD19 (1:200; cat. no. ab134114), CD38 (1:200; cat. no. ab108403), CD3 (1:30; cat. no. ab16669), Bcl-6 (undiluted; cat. no. ab172610) and Bcl-2 (1:100; cat. no. ab32124), which were purchased from Abcam (Cambridge, UK); MPO (1:50; cat.no. TC66701-2), CD5 (1:100; cat. no. M364101-2), CD38 (1:50; cat. no. TC67401-2), CD56 (1:50; cat. no. TC67401-2), CD4 (1:10; cat. no. FR86850-2), CD8 (1:10; cat. no. F076501-2), CD23 (1:50; cat. no. IS78130-2), Kappa (1:2,500; cat. no. A019102-2) and Lambda (1:2,500; cat. no. 239222), which were purchased from Dako; Agilent Technologies, Inc. (Santa Clara, CA, USA); CD20 (1:100; cat. no. MA513141), CD79a (1:100; cat. no. 186490) and MIB-1 (1:100; cat. no. PA5-16446), which were purchased from Zymed; TDT (undiluted; cat. no. PL0403179) and CyclinD1 (1:50; cat. no. or677046), which were purchased from Neomarkers (https://univ.biomart.cn/). After washing with PBS, the tissue sections were placed to react with biotin-labeled rabbit anti-guinea pig IgG secondary antibody (1:1,000; cat. no. QN2726; Beijing Baiaoleboke Co., Ltd., Beijing, China) at $37^{\circ} \mathrm{C}$ for $30 \mathrm{~min}$. The sections were washed with PBS for $5 \mathrm{~min}$, repeated three times, then incubated with VECTA STAIN Elite ABC standard kit (Vector Laboratories, Inc., Burlingame, CA, USA) according to the manufacturer's protocol at $37^{\circ} \mathrm{C}$ for $30 \mathrm{~min}$, finally the signals were visualized with a chromogenic kit (Vector Laboratories, Inc.) according to the manufacturer's protocol or by microscopic examination: Optical microscope (Olympus BX41, Olympus Corporation, Tokyo, Japan) was used, and x4, x10 and x40 objectives were used for observation, respectively.

Data acquisition included the following aspects: i) Basic information; ii) diagnostic information; iii) clinical manifestations; iv) imaging manifestations; v) histopathology and laboratory examination indices; vi) treatment situations; vii) Eastern Cooperative Oncology Group (ECOG) score (22), and; viii) curative effect.

Treatment regimens and treatment response assessment. The assessment of the treatment response was based on the consensus standards developed by the International Primary CNS Lymphoma Collaborative Group (IPCG) (23), consist of complete response (CR), partial response (PR), progressive disease (PD), and stable disease (SD). Overall response (OR) includes PR and CR. The summary of the assessment criteria is presented in Table I. The follow-up deadline was December 31, 2015.

There were XX courses of treatment. In each course of treatment, the total dose of MTX was $3.0 \mathrm{~g} / \mathrm{m}^{2}$, while the total dose of $\mathrm{Vm} 26$ was $300 \mathrm{mg}$, while the total dose of Vm26 in every course of treatment was $300 \mathrm{mg}$. During the intravenous administration of MTX, patients were required to undergo hydration and alkalization, and to receive leucovorin rescue. The interval between chemotherapy sessions was three weeks. Treatment regimens were altered if patients suffered from disease progression, or if unacceptable toxic reactions occurred.

Statistical analysis. The collected data were analyzed using SPSS 20.0 software (IMB Corp., Armonk, NY, USA). Overall survival (OS) time was defined as the period from the time the patient was diagnosed to the time the patient succumbed, or up to the follow-up deadline. Progression-free survival (PFS) was defined as the period from the time the patient was diagnosed to disease recurrence/progression or last point of contact with the patient. Univariate analysis was conducted using a log-rank test, survival analysis was conducted using Kaplan-Meier curves and log-rank test and multivariate analysis was conducted using a Cox's regression. $\mathrm{P}<0.05$ was considered to indicate a statistically significant difference.

\section{Results}

Patients. The cohort of 56 patients comprised 38 males and 18 females. The age of these patients ranged between 
25-74 years, with a median age of 54.5 years. A total of 13 patients $(23.21 \%)$ were $\geq 60$ years.

\section{Clinical characteristics of patients}

Clinical symptoms. The common symptoms of PCNSL include elevated intracranial pressure $(53.6 \%)$, somatic dysfunction (53.6\%), cognitive dysfunction (33.9\%), impaired vision (30.3\%) and language disorder (17.9\%).

Imaging tests. All patients underwent cranial magnetic resonance imaging, and 17 additionally underwent positron emission tomography-computed tomography. Patients with multiple lesions accounted for $60.7 \%$ of the total cohort, and patients with deep lesions accounted for $60.7 \%$. The frontal lobe was the most common lesion site, accounting for $46.4 \%$ patients, followed by the parietal lobe $(25 \%)$ and basal ganglia (23.2\%).

ECOG score results. A total of 12 patients received 1 point, 13 patients received 2 points, 26 patients received 2 points, 4 patients received 3 points and 2 patients received 5 points. Patients who received a score within 1-3 points accounted for $91 \%$ of the total cohort.

\section{Histopathology and laboratory tests}

Histopathology. The histopathology of all 56 patients was diffuse large B cell lymphoma.

Cerebrospinal fluid examination. Cerebrospinal fluid examination was performed on 26 patients. Among 26 patients, a Pandy's test exhibited a positive result in 19 patients (73.1\%), the protein content increased in 18 patients (69.1\%), and spinal cord involvement, such as chest-back or lower-back pain, weakness of lower limbs, paraplegia and superficial sensory dullness or disappearance, occurred in 11 patients (42.3\%). Spinal cord involvement: i) The patients had periodical lumbar pain and compression symptoms; ii) Enhanced MRI revealed lesions; iii) The lesions shrunk after treatment.

Serum $L D H$ and serum and urinary $\beta 2$-microglobulin $(M G)$ examination. Serum LDH (normal range from 109 to $245 \mathrm{U} / \mathrm{l}$ ) was increased in 21 patients (37.5\%), serum $\beta 2-\mathrm{MG}$ (normal range from 1 to $2.8 \mu \mathrm{g} / \mathrm{ml}$ ) was increased in 32 patients (57.1\%), and urinary $\beta 2-\mathrm{MG}$ (normal range from 0 to $0.3 \mu \mathrm{g} / \mathrm{ml}$ ) was increased in 21 patients $(37.5 \%)$.

\section{Treatment protocol}

Treatment regimen. Treatment regimens were divided into three categories: i) Radiotherapy + chemotherapy (radiotherapy followed by chemotherapy); ii) chemotherapy + radiotherapy (chemotherapy followed by radiotherapy); iii) chemotherapy alone. All had been done. 7 patients received radiotherapy + chemotherapy, 20 patients received chemotherapy + radiotherapy, and 29 patients received chemotherapy alone. MTX group: 3 patients received radiotherapy + chemotherapy, 13 patients received chemotherapy + radiotherapy, and 13 patients received chemotherapy alone.

The 56 patients in the present study all received chemotherapy regimens with HD-MTX. Among these patients, $51.8 \%$ patients were treated with chemotherapy alone, and the remaining $48.2 \%$ patients were treated with chemotherapy and radiotherapy. The majority of the patients received radiotherapy followed by chemotherapy; only 7 patients $(12.5 \%)$ received chemotherapy followed by radiotherapy. These results revealed that the median PFS was 20 months and the median OS time was 31 months in patients who received chemotherapy alone. The median PFS and median OS were 21 and 37 months, respectively, in patients who received chemotherapy followed by radiotherapy; while median PFS and median OS were 22 and 52 months, respectively, in patients who received radiotherapy followed by chemotherapy. Between patients who received chemotherapy alone and the patients who received chemotherapy combined with radiotherapy, the difference in PFS was not statistically significant.

\section{Curative effect analysis}

Treatment response. The evaluation of disease progression was performed following three courses of chemotherapy for all patients. In the MTX+Vm26 group, 12 patients (42.85\%) achieved CR, 10 patients (35.71\%) achieved PR and the OR rate was $78.57 \%$. In the MTX group, 7 patients (25\%) achieved CR, 11 patients $(39.29 \%)$ achieved PR and the OR rate was 64.3\%. Differences in CR and OR between these two groups were not statistically significant (Table II).

PFS and OS. At the end of the follow-up, 26 patients succumbed, 29 patients survived, and 1 patient in the MTX+Vm26 group was lost to follow-up subsequent to confirmation of relapse. The median PFS were 22 and 12 months in the MTX+Vm26 and MTX groups, respectively; the median OS rates were 57 and 28 months, respectively. These two indices were significantly increased in the MTX+Vm26 group compared with the MTX group $(\mathrm{P}<0.05$; Table II). The median OS was 40 months in 55 patients (total patient cohort minus 1 patient lost to follow up) (Fig. 1A). Comparison of the median OS between the two treatment groups revealed that the OS of the MTX+Vm26 group was significantly increased compared with the MTX group (Fig. 1B).

Mortality. Overall, mortality was $47.3 \%$ at the end of follow-up, in which 13 patients $(47.3 \%)$ were from the MTX group and 13 patients (48.1\%) were from the MTX+Vm26 group, and the difference between the mortality rates between the two treatment groups was not statistically significant $(\mathrm{P}=0.898)$.

Disease progression. A total of 30 patients exhibited disease progression at the end of the follow-up, in which 16 patients (57.14\%) were from the MTX group and 14 patients (50\%) were from the MTX+Vm26 group; the difference between these two groups was not statistically significant $(\mathrm{P}=0.592)$.

\section{Prognosis analysis}

Univariate analysis. Univariate analysis was performed to analyze factors that may be associated with the prognosis of disease. The disease progression curve was evaluated using the Kaplan-Meier method and the factors were compared using a log-rank test. Detailed results are indicated in Table III. Univariate analysis revealed that age was a prognostic factor in patients with PCNSL, and the median PFS of patients with an age $>60$ years was 10 months shorter compared with that in 
Table I. Criteria for assessment of therapeutic effects.

\begin{tabular}{lllll}
\hline $\begin{array}{l}\text { Treatment } \\
\text { response }\end{array}$ & \multicolumn{1}{c}{$\begin{array}{c}\text { Imaging } \\
\text { examination }\end{array}$} & $\begin{array}{c}\text { Dosage of } \\
\text { glucocorticoid }\end{array}$ & \multicolumn{1}{c}{$\begin{array}{c}\text { Ophthalmologic } \\
\text { examination }\end{array}$} & $\begin{array}{c}\text { Cerebrospinal } \\
\text { fluid examination }\end{array}$ \\
\hline $\mathrm{CR}$ & No enhancement & Nil & Normal & Negative \\
$\mathrm{CRu}$ & No enhancement & Any & Normal & Negative \\
& No abnormal lesions & Any & Micro RPE was abnormal & Negative \\
PR & $\begin{array}{l}\text { Enhanced lesion was } \\
\text { reduced by 50\% }\end{array}$ & No association & Micro RPE was abnormal or & Negative \\
& No enhancement & No association & Vormal & Vitreous cell or retinal infiltration, Persistent positive or suspicious \\
& & & reduction & positive \\
& Tumor was reduced by 25\% & No association & Recurrence or new eye lesions & Recurrence or positive
\end{tabular}

$\mathrm{CR}$, complete remission; Cru, unconfirmed complete remission; RPE, retinal pigment epithelial cells; PR, partial remission; PD, disease progression: $\mathrm{SD}$, stable disease, in a state between PR and PD. In the present study, CR included CR and CRu.

Table II. Comparison of the curative effects between treatments groups.

\begin{tabular}{|c|c|c|c|c|}
\hline Group & $\mathrm{MTX}+\mathrm{Vm} 26$ & MTX & $\chi^{2}$ & P-value \\
\hline CR & $12(42.85 \%)$ & $7(25 \%)$ & 1.991 & 0.158 \\
\hline PR & $10(35.71 \%)$ & $11(39.29 \%)$ & 0.076 & 0.783 \\
\hline SD & $4(14.29 \%)$ & $4(14.29 \%)$ & 0.000 & 1.000 \\
\hline PD & $2(7.14 \%)$ & $6(21.43 \%)$ & 1.313 & 0.252 \\
\hline OR & $22(78.57 \%)$ & $18(64.3 \%)$ & 1.400 & 0.237 \\
\hline Median PFS, months & 22 & 12 & 5.462 & 0.019 \\
\hline Median OS, months & 57 & 28 & 6.196 & 0.013 \\
\hline
\end{tabular}

$\mathrm{CR}$, complete response; PR, partial remission; SD, stable disease; PD, progressive disease; OR, overall response; PFS, progression-free survival; OS, overall survival; MTX, methotrexate; Vm26, Teniposide.

patients with an age of $\leq 60$ years (Fig. 1C). These are consistent with the results of previous studies (24-26). The median PFS was 69 months in patients who achieved CR following three courses of treatment, and was 20 months in patients who did not achieve CR. The difference between these two was marked, but was not statistically significant $(\mathrm{P}=0.061)$. The median PFS was 12 months in the MTX group, and 22 months in the HD-MTX+Vm26 group, and the difference between these two groups was statistically significant ( $\mathrm{P}=0.033$; Fig. 1D).

Multivariate analysis. As identified from the univariate analysis, those progression-associated factors with a $\mathrm{P}<0.05$, and factors with a $\mathrm{P}>0.05$ with a PFS presenting a difference, were analyzed using multivariate analysis. These results revealed that age and treatment regimen were independent risk factors that affect the progression of PCNSL (Table IV).

Toxic reaction. A total of 8 patients (28.57\%) in the Vm26+MTX group and 6 patients $(21.43 \%)$ in the MTX group exhibited grade $3 / 4$ hematologic leukopenia. A total of 5 patients in the MTX+Vm26 group (17.86\%) and 3 patients in the MTX group (10.71\%) exhibited agranulocytosis and fever. A total of 7 patients $(25 \%)$ developed pneumonia. In addition, 5 patients in the MTX+Vm26 (17.86\%) and 4 patients in the MTX group (14.29\%) exhibited grade 2 liver function impairment (Table V). No patients exhibited $\geq$ grade 3 hyperpyrexia and cardiac toxicity.

\section{Discussion}

PCNSL is a rare type of extra-nodal lymphoma. The initial symptoms include changes in cognitive function, increased intracranial pressure and hemiplegia. During the early stage, lesions are confined to the CNS; and in the late stage, $<10 \%$ of patients exhibit tumors that spread outside the CNS (27). Although the cancer cells originates from lymphocytes, PCNSL usually is considered as a brain tumor in practice due to its therapeutic challenges, such as drug delivery through blood-brain barrier, and cerebral toxicity (28). Due to these unique characteristics of the clinical symptoms and biological behaviors of the tumor cells in PCNSL, the 2008 WHO Hematopoietic and Lymphoid Classification lists it as an independent disease entity (21). In the past three decades, epidemiological data in Western countries, such as Norway and Sweden revealed that the incidence of PCNSL continues to increase (29-31). The prognosis of patients with PCNSL is relatively poor, and the prognosis of patients with HIV 
A
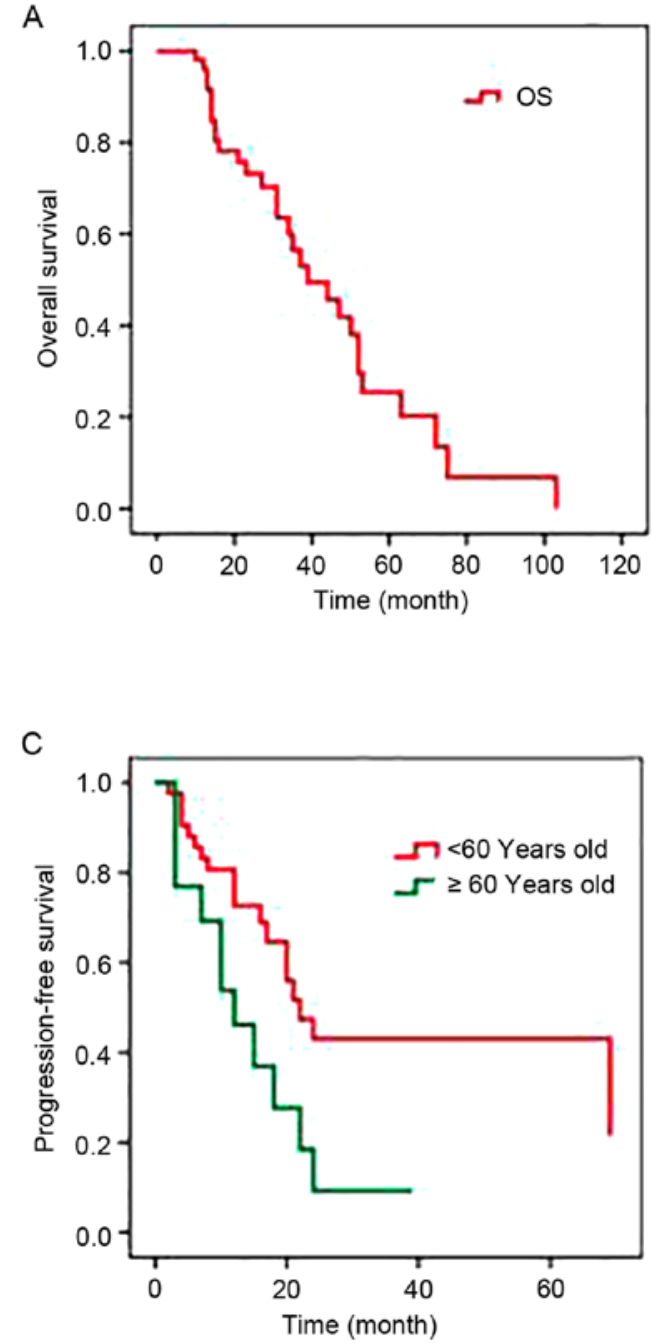
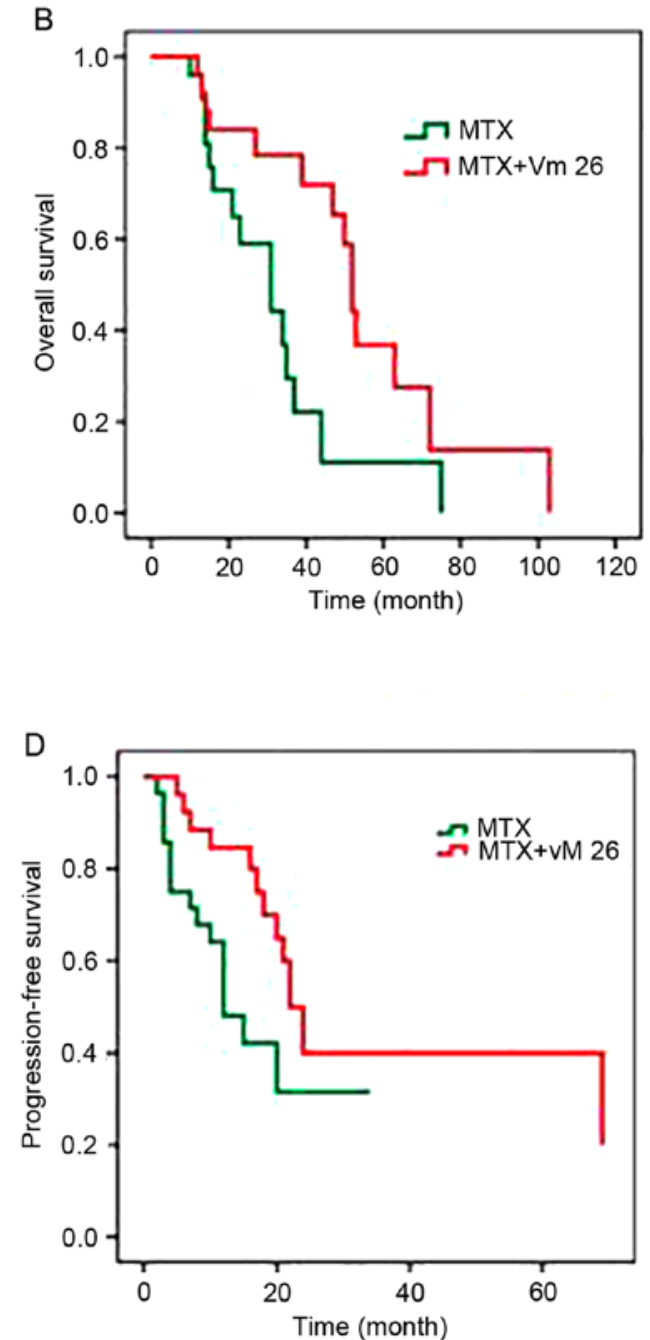

Figure 1. Median OS and PFS. (A) Median OS in 55 patients. (B) Median OS between the two groups. (C) The association between PFS and age. (D) The association between PFS and courses of treatment. OS, overall survival; PFS, progression-free survival. MTX, methotrexate; Vm26, Teniposide.

infection is particularly poor (32). In the present study, no patient had HIV infection.

Statistical data from the United States of America between 2000-2004 revealed that the average age of patients diagnosed with PCNSL was 60 years, and the age of the majority of patients ranged within 50-65 years $(5,33,34)$. A study conducted in Spain revealed that within the period 1990-2010, the average age of patients diagnosed with PCNSL was 59.3 years (35). In the present study, the average age of patients was 53.5 years, which was decreased compared with that in other countries.

PCNSL exhibits three pathological types: DLBCL, T cell lymphoma and other types of B cell lymphoma. DLBCL accounts for $>90 \%$ of PCNSL (36). The pathological types of the 56 patients were all DLBCL in the present study.

The clinical symptoms of patients with PCNSL were associated with the location of tumor lesions $(37,38)$. A study in Spain revealed that patients with cognitive function decline accounted for $33.4 \%$, and those with cephalea accounted for $25 \%$ (35). The present study revealed that increased intracranial pressure and physical movement disorders were common, and the rates for the two symptoms were $53.6 \%$; followed by cognitive dysfunction (33.9\%), vision impairment (30.3\%) and language disorder (17.9\%).
Statistics from the guidelines of the National Comprehensive Cancer Network (NCCN) revealed that $>90 \%$ of patients with PCNSL exhibited lesions affecting the brain parenchyma, and over one-half of these patients possessed multiple lesions (39). Fraser et al (40) demonstrated that $35 \%$ of patients with PCNSL exhibited multiple lesions, and up to $38 \%$ of patients exhibited lesions in the cerebral hemispheres. In the present study, frontal lobe involvement $(46.4 \%)$ accounted for the largest proportion of lesion sites. The proportion of patients with deep involvement was $60.7 \%$. The majority of patients exhibited multiple lesions $(60.7 \%)$.

The physical fitness score of patients was one of the factors that affected the choice of treatment regimens. It was not recommended that patients with poor physical condition use the HD-MTX+Vm26 regimen (41). In the present study, the ECOG scores of patients were concentrated within 1-3 points.

Results of the cerebrospinal fluid examination in 26 patients indicated that the Pandy's test demonstrated a positive result in 19 patients $(73.1 \%)$, and that the protein level in cerebrospinal fluid was increased in 18 patients (not examined using a Pandy's test) (69.2\%). The examinations of serum LDH, serum $\beta 2-\mathrm{MG}$ and urine $\beta 2-\mathrm{MG}$ revealed that $37.5 \%$ of patients exhibited increased serum LDH, $57.1 \%$ of patients exhibited 
Table III. Univariate analysis.

\begin{tabular}{|c|c|c|c|c|}
\hline Potential prognostic factors & $\mathrm{PD} /$ total cases & Median PFS, months & $\chi^{2}$ & P-value \\
\hline Age, years & & 20 & 6.021 & 0.014 \\
\hline$\leq 60$ & $19 / 43$ & 22 & & \\
\hline$>60$ & $11 / 13$ & 12 & & \\
\hline ECOG score, points & & & 0.017 & 0.896 \\
\hline $0-1$ & $6 / 12$ & 22 & & \\
\hline$\geq 1$ & $24 / 44$ & 20 & & \\
\hline Deep lesions accumulating & & & 1.117 & 0.291 \\
\hline No & $14 / 22$ & 16 & & \\
\hline Yes & $16 / 34$ & 20 & & \\
\hline Number of lesions & & & 0.741 & 0.389 \\
\hline Single & $14 / 22$ & 16 & & \\
\hline Multiple & $16 / 34$ & 20 & & \\
\hline Serum LDH & & & 1.100 & 0.294 \\
\hline Normal (106-211 U/l) & $15 / 35$ & 24 & & \\
\hline Abnormally increasing & $15 / 21$ & 18 & & \\
\hline Blood $\beta_{2}-\mathrm{MG}$ & & & 0.793 & 0.373 \\
\hline Normal $(0.70-1.80 \mathrm{mg} / \mathrm{l})$ & $14 / 24$ & 17 & & \\
\hline Abnormally increasing & $16 / 32$ & 22 & & \\
\hline Urine $\beta_{2}-\mathrm{MG}$ & & & 0.076 & 0.783 \\
\hline Normal $(<0.25 \mathrm{mg} / \mathrm{l})$ & $19 / 35$ & 18 & & \\
\hline Abnormally increasing & $11 / 21$ & 21 & & \\
\hline CSF; Pandy's test & & & 1.670 & 0.196 \\
\hline Negative & $4 / 6$ & 10 & & \\
\hline Positive & $13 / 20$ & 20 & & \\
\hline CSF protein quantification & & & 0.948 & 0.330 \\
\hline Normal $(150-450 \mathrm{mg} / \mathrm{l})$ & $5 / 8$ & 10 & & \\
\hline Abnormally increasing & $12 / 18$ & 20 & & \\
\hline CSF glucose level & & & 0.160 & 0.689 \\
\hline Normal $(2.5-4.4 \mathrm{mmol} / \mathrm{l})$ & $12 / 20$ & 20 & & \\
\hline Abnormal & $5 / 6$ & 20 & & \\
\hline CSF chloride & & & 0.915 & 0.339 \\
\hline Normal $(2.7-3.9 \mathrm{mmol} / \mathrm{l})$ & $12 / 14$ & 20 & & \\
\hline Abnormal & $5 / 12$ & 22 & & \\
\hline CSF white blood cell count & & & 2.296 & 0.130 \\
\hline Normal $\left(0-5 / \mathrm{mm}^{3}\right)$ & $12 / 19$ & 24 & & \\
\hline Abnormally increasing & $5 / 7$ & 20 & & \\
\hline Lesion resection by surgery & & & 0.562 & 0.454 \\
\hline Yes & $12 / 31$ & 20 & & \\
\hline No & $18 / 25$ & 22 & & \\
\hline Received CR & & & 3.519 & 0.061 \\
\hline No & $22 / 37$ & 20 & & \\
\hline Yes & $8 / 19$ & 69 & & \\
\hline Chemotherapy regiments & & & 4.557 & 0.033 \\
\hline HD-MTX & $16 / 28$ & 12 & & \\
\hline HD-MTX + Vm26 & $14 / 28$ & 22 & 0.920 & 0.631 \\
\hline \multicolumn{5}{|l|}{ Therapeutic schedule } \\
\hline Chemotherapy & $16 / 29$ & 18 & & \\
\hline Chemotherapy + radiotherapy & $4 / 7$ & 21 & & \\
\hline Radiotherapy + chemotherapy & $10 / 20$ & 22 & & \\
\hline
\end{tabular}

ECOG, Eastern Cooperative Oncology Group; PFS, progression-free survival; LDH, lactate dehydrogenase; $\beta_{2}$-MG, $\beta_{2}$-microglobulin; PD, progressive disease; CSF, cerebrospinal fluid; HD-MTX, high-dose methotrexate; Vm26, Teniposide 
Table IV. Multivariate analysis.

\begin{tabular}{lcccccrr}
\hline Factor & B & SE & Wald test & D.f. & P-value & Exp (B) & $95 \%$ CI \\
\hline Age & -0.886 & 0.400 & 4.902 & 1 & 0.027 & 0.412 & $0.188-0.903$ \\
Treatment remission & 0.595 & 0.428 & 1.928 & 1 & 0.165 & 1.812 & $0.783-4.195$ \\
Treatment regimen & 0.951 & 0.412 & 5.321 & 1 & 0.021 & 2.587 & $1.154-5.803$ \\
\hline
\end{tabular}

B, regression coefficient estimate value; SE, standard error; D.f, degrees of freedom; Exp (B), odds ratio of the outcome; CI, confidence interval.

Table V. Comparison of toxic reactions between MTX + Vm26 and MTX treatment groups.

\begin{tabular}{|c|c|c|c|c|}
\hline Toxicity & $\mathrm{MTX}+\mathrm{Vm} 26(\%)$ & $\operatorname{MTX}(\%)$ & $\chi^{2}$ & P-value \\
\hline \multicolumn{5}{|l|}{ Hematologic } \\
\hline Grade 3-4 leukopenia & $8(28.57)$ & $6(21.43)$ & 0.381 & 0.537 \\
\hline Grade 3-4 anemia & $3(10.71)$ & $2(7.14)$ & 0.220 & 0.639 \\
\hline Grade 3-4 PLT decrease & $4(14.29)$ & $2(7.14)$ & 0.747 & 0.388 \\
\hline \multicolumn{5}{|l|}{ Non-hematologic } \\
\hline Grade 2 abnormal liver function & $5(17.86)$ & $4(14.29)$ & 0.132 & 0.716 \\
\hline Grade 2 renal function damage & $4(14.29)$ & $5(17.86)$ & 0.132 & 0.716 \\
\hline Grade 2 numbness & $3(10.71)$ & $2(7.14)$ & 0.220 & 0.639 \\
\hline
\end{tabular}

PLT, platelet; MTX, methotrexate; Vm26, Teniposide.

increased serum $\beta 2-\mathrm{MG}$ and $37.5 \%$ of patients exhibited increased urinary $\beta 2-\mathrm{MG}$.

The feasibility and effectiveness of HD-MTX in the treatment of PCNSL have been recognized. It may be used for induction therapy and salvage therapy (1). Results of a study conducted by Prica et al (42) revealed that compared with chemotherapy alone, chemotherapy combined with radiotherapy was an improved induction regimen in younger patients (aged $<60$ years). However, due to toxic side effects, it is recommended that elderly patients ( $>60$ years old) should receive chemotherapy alone $(43,44)$. A study conducted by Muirhead et al (45) revealed that for patients who were not able to tolerate large-dose chemotherapy, 3-year survival rates of salvage radiotherapy were increased compared with that of the second-line chemotherapy (60 vs. 33\%). Salvage radiotherapy may be a choice for young patients with recurrent/refractory PCNSL (7). The use of chemotherapy or radiotherapy should be determined on an individual patient basis. The 56 patients in the present study all received chemotherapy regimens with HD-MTX. Among these patients, $51.8 \%$ patients were treated with chemotherapy alone, and the remaining $48.2 \%$ patients were treated with chemotherapy and radiotherapy. Between patients who received chemotherapy alone and the patients who received chemotherapy combined with radiotherapy, the difference in PFS was not statistically significant. Additionally, whilst the difference in median OS was notable, it was not statistically significant $(\mathrm{P}=0.24)$. This may be associated with not enough sample size between chemotherapy alone group and chemotherapy combined with radiotherapy group.
Total cranial irradiation may cause toxic side effects, such as the decrease in cognitive function. Ongoing clinical trials are being conducted on replacing radiotherapy alone, with combined chemotherapy (46). On the basis of HD-MTX, these regimens contain 1-3 chemotherapeutic drugs with different mechanisms, such as HD-Ara-C, temozolomide, rituximab, idarubicin and etoposide (12). Vm26 is a type of semi-synthetic derivative of podophyllotoxin, which has a high liposolubility and may effectively pass through the blood-brain barrier (19). However, it remains unknown whether this drug, in combination with HD-MTX, may improve the curative effect in the treatment of PCNSL.

In the present study, in the HD-MTX+Vm26 group, 12 patients $(42.85 \%)$ achieved CR and 10 patients $(35.71 \%)$ achieved PR, while in the HD-MTX group, 7 patients $(25 \%)$ achieved CR, 11 patients $(39.29 \%)$ achieved PR, and the OR rate was $64.3 \%$. The difference of CR and OR between these two groups was notable, but not statistically significant. This may be associated with the small number of cases in the two groups. The median PFS was 22 months in the HD-MTX+Vm 26 group and 12 months in the HD-MTX group, and the difference between the two groups was statistically significant $(\mathrm{P}=0.019)$. The median OS was 57 months in the HD-MTX+Vm26 group and 28 months in the HD-MTX group $(\mathrm{P}=0.013)$. This suggests that HD-MTX combined with Vm26 may effectively improve the long-term curative effect of the treatment of patients with PCNSL.

Ferreri et al (33) conducted a multicenter retrospective study. Their results revealed 5 factors that were associated with poor prognosis: i) Age $>60$ years old; ii) ECOG grades 2-4; 
iii) increased serum LDH; iv) increased protein level in cerebrospinal fluid; and v) tumors located in the deep areas of brain tissues, including periventricular tissues, basal ganglia, brainstem and cerebellum. Hu et al (47) demonstrated that solitary or multiple tumor lesions were independent factors that affected prognosis. Abrey et al (48) indicated that only age and physical condition were of significance for prognosis.

Results of the associated prognostic factors in the present study revealed that age was an independent prognostic factor in patients with PCNSL in the univariate and multivariate analyses. The time to disease progression in patients with an age of $>60$ years was $\sim 10$ months shorter compared with that in patients with an age of $\leq 60$ years. This was consistent with the results of the majority of studies conducted outside China (33). In addition, the present study revealed that the difference in chemotherapy regimen is also an independent prognostic factor. Compared with HD-MTX alone, the HD-MTX+Vm26 regimen was not only useful in improving the $\mathrm{CR}$ rate for its short-term curative effect (three courses), it was also able to improve its long-term curative effect.

The present study revealed that the physical fitness scores were not associated with prognosis. This may be due to the small sample size in the present study. In addition, early studies conducted in countries outside China primarily focused on the association between physical fitness score and OS. However, the present study focused on PFS duration. This may also lead to different conclusions.

A previous study revealed that serum LDH level was an independent prognostic factor for PCNSL: The prognosis of patients with increased serum LDH level was poor (33). Results of the present study revealed that serum LDH level exhibited no significant effect on PFS in patients. Although there have been multiple studies on the association between the survival time and the serum and urinary $\beta 2-\mathrm{MG}$ levels in patients with PCNSL worldwide, no consensus has been obtained (49). The results of the present study revealed that increased serum and urinary $\beta 2-M G$ levels did not significantly affect the PFS of patients.

Increased protein levels in the cerebrospinal fluid in patients with PCNSL suggest that these lesions may have affected the cerebral pia mater (24). However, it may also be induced by increased tumor load. These two factors may lead to poor prognosis in patients (33). The present study revealed that the increase in cerebrospinal fluid protein level led to a significant difference in PFS, which were 20 and 10 months, respectively. However, this is probably due to the small sample size, and the difference was not statistically significant.

Results of a study conducted by Ferreri et al (33) revealed that the 2-year survival rate of patients with PCNSL who did not exhibit deep structure involvement was $\sim 42 \%$, and that of patients who demonstrated deep structure involvement was $28 \%$. In the present study, $60.7 \%$ of patients exhibited lesions that affected the deep structures. Furthermore, statistical data revealed that this had no significant association with the PFS of patients.

A previous study suggested that surgery did not confer significant benefits to patients with PCNSL, and had risk of damage to neural functions (28). Surgical removal is not recommended in the majority of cases (27). Cases of surgical operation in patients with PCNSL were few, and little recent publications have demonstrated that surgical resection could be established as a prognostic factor in PCNSL $(32,37)$. In 2012, the results of a German PCNSL SG-1 trial revealed that surgical removal may extend the PFS of patients (50-52). Fraser et al (40) considered that surgical removal was advantageous to rapidly relieving symptoms and decreasing glucocorticoid dose, which eliminated the production of drug-resistant tumor clones. In addition, due to modern surgical positioning technology, in 2012 a German primary central nervous system lymphoma (PCNSL) SG-1 test revealed that, surgical resection of PCNSL tumor lesions can prolong the progression free survival time of patients $(53,54)$. The results of the present study revealed that median PFS was 20 months in patients who underwent the surgical removal of lesions, while PFS was 22 months in patients who underwent directional puncture biopsy. The difference was not statistically significant between these two surgical methods.

A previous study identified that patients with multiple lesions exhibited shorter survival times (24), but this was not recognized. An early study revealed that the number of lesions would affect the survival time of patients, and the survival time of patients with a large number of lesions is relatively shorter (42). However, this research result has not been recognized. In the present study, univariate analysis of the number of lesions also revealed that the difference in PFS between patients with multiple lesions and patients with single lesion was not statistically significant.

In the present study, the univariate analysis on the number of lesions revealed that the difference in PFS between patients with multiple lesions and patients with a single lesion was not statistically significant.

In summary, the results of the present study revealed that age and the treatment regimen were independent prognostic factors of PCNSL. Compared with patients $>60$ years old, patients who were $\leq 60$ years old exhibited a significantly prolonged PFS. At the same time, the PFS of patients who received the combined treatment of Vm25+HD-MTX was significantly prolonged when comparing with HD-MTX alone treatment. The difference in the toxic reaction of chemotherapy was not statistically significant. However, the sample size of the present study was small, and this single-center retrospective study has limitations. Therefore, the conclusions of the present study should be confirmed through prospective multicenter clinical trials in the future.

The clinical data of the 56 patients with PCNSL were retrospectively analyzed. It was revealed that: i) Age is an independent prognostic factor in patients with PCNSL, in which patients who were $\leq 60$ years old exhibited longer PFS compared with patients who were $>60$ year old; ii) the median PFS time was 69 months in patients who achieved CR following three courses of treatment and 20 months in patients who did not achieve remission, and the difference between these two was marked but not statistically significant $(\mathrm{P}=0.061)$; iii) PFS time was 22 months in the HD-MTX+Vm26 group and was 12 months in the MTX group, and median OS time was 57 months in the HD-MTX+Vm26 group and was 28 months in the MTX group. Both PFS time and median OS time were significantly different between these two groups. Therefore, compared with HD-MTX alone, the combined treatment of HD-MTX+Vm26 exhibited a significantly improved long-term curative effect in the treatment of PCNSL. 


\section{Acknowledgements}

Not applicable.

\section{Funding}

No funding was received.

\section{Availability of data and materials}

The datasets used and/or analyzed during the current study are available from the corresponding author on reasonable request.

\section{Authors' contributions}

YXW contributed to the conception and design of the study and drafted the manuscript. YXW, YH, XPX, BBC, ZGL, YM, TLD and QW acquired, analyzed and interpreted the data and revised the manuscript critically for important intellectual content. All authors read and approved the final version of the manuscript.

\section{Ethics approval and consent to participate}

This study was conducted in accordance with the declaration of Helsinki. This study was conducted with approval from the Ethics Committee of Huashan Hospital Affiliated to Fudan University. Written informed consent was obtained from the participants.

\section{Patient consent for publication}

Not applicable.

\section{Competing interests}

The authors declare that they have no competing interests.

\section{References}

1. Ferreri AJ, Abrey LE, Blay JY, Borisch B, Hochman J Neuwelt EA, Yahalom J, Zucca E, Cavalli F, Armitage J and Batchelor T: Summary statement on primary central nervous system lymphomas from the Eighth International Conference on Malignant Lymphoma, Lugano, Switzerland, June 12 to 15, 2002. J Clin Oncol 21: 2407-2414, 2003.

2. Miller DC, Hochberg FH, Harris NL, Gruber ML, Louis DN and Cohen H: Pathology with clinical correlations of primary central nervous system non-Hodgkin's lymphoma. The Massachusetts General Hospital experience 1958-1989. Cancer 74: 1383-1397, 1994.

3. Rubenstein JL, Hsi ED, Johnson JL, Jung SH, Nakashima MO, Grant B, Cheson BD and Kaplan LD: Intensive chemotherapy and immunotherapy in patients with newly diagnosed primary CNS lymphoma: CALGB 50202 (Alliance 50202). J Clin Oncol 31: 3061-3068, 2013.

4. Jang JE, Kim YR, Kim SJ, Cho H, Chung H, Lee JY, Park H, Kim Y, Cheong JW, Min YH and Kim JS: A new prognostic model using absolute lymphocyte count in patients with primary central nervous system lymphoma. Eur J Cancer 57: 127-135, 2016.

5. Joerger M, Huitema AD, Krähenbühl S, Schellens JH, Cerny T, Reni M, Zucca E, Cavalli F and Ferreri AJ: Methotrexate area under the curve is an important outcome predictor in patients with primary CNS lymphoma: A pharmacokinetic-pharmacodynamic analysis from the IELSG no. 20 trial. Br J Cancer 102: 673-677, 2010 .
6. Hottinger AF, Alentorn A and Hoang-Xuan K: Recent developments and controversies in primary central nervous system lymphoma. Curr Opin Oncol 27: 496-501, 2015.

7. Hoang-Xuan K, Bessell E, Bromberg J, Hottinger AF, Preusser M, Rudà R, Schlegel U, Siegal T, Soussain C, Abacioglu U, et al: Diagnosis and treatment of primary CNS lymphoma in immunocompetent patients: Guidelines from the European Association for Neuro-Oncology. Lancet Oncol 16: e322-e332, 2015.

8. Bhojwani D, Sabin ND, Pei D, Yang JJ, Khan RB, Panetta JC, Krull KR, Inaba H, Rubnitz JE, Metzger ML, et al: Methotrexate-induced neurotoxicity and leukoencephalopathy in childhood acute lymphoblastic leukemia. J Clin Oncol 32: 949-959, 2014.

9. Cohen IJ: Neurotoxicity after high-dose methotrexate (MTX) is adequately explained by insufficient folinic acid rescue. Cancer Chemother Pharmacol 79: 1057-1065, 2017.

10. Cher L, Glass J, Harsh GR and Hochberg FH: Therapy of primary CNS lymphoma with methotrexate-based chemotherapy and deferred radiotherapy: Preliminary results. Neurology 46: 1757-1759, 1996.

11. Guha-Thakurta N, Damek D, Pollack C and Hochberg FH: Intravenous methotrexate as initial treatment for primary central nervous system lymphoma: Response to therapy and quality of life of patients. J Neurooncol 43: 259-268, 1999.

12. Citterio G, Reni M and Ferreri AJ: Present and future treatment options for primary CNS lymphoma. Expert Opin Pharmacother 16: 2569-2579, 2015.

13. Ferreri AJ, Reni M, Foppoli M, Martelli M, Pangalis GA, Frezzato M, Cabras MG, Fabbri A, Corazzelli G, Ilariucci F, et al: High-dose cytarabine plus high-dose methotrexate versus high-dose methotrexate alone in patients with primary CNS lymphoma: A randomised phase 2 trial. Lancet 374: 1512-1520, 2009.

14. Crivellari D, Lombardi D, Spazzapan S, Veronesi A and Toffoli G: New oral drugs in older patients: A review of idarubicin in elderly patients. Crit Rev Oncol Hematol 49: 153-163, 2004.

15. Geffen DB and Man S: New drugs for the treatment of cancer, 1990-2001. Isr Med Assoc J 4: 1124-1131, 2002.

16. Xu G, Yang M, Tong H, Mao L and Jin J: High-dose methotrexate plus temozolomide as a salvage treatment in metastatic central nervous system Lymphoma: Two cases report and review of literature. Int J Clin Exp Med 8: 8222-8225, 2015.

17. Ferreri AJ, Cwynarski K, Pulczynski E, et al: The addition of thiotepa and rituximab to antimetabolites significantly improves outcome in primary CNS lymphoma: The first randomization of the IELSG-32 trial. Int Conference Malignant Lymphoma, 2015.

18. Ponzoni M, Issa S, Batchelor TT and Rubenstein JL: Beyond high-dose methotrexate and brain radiotherapy: Novel targets and agents for primary CNS lymphoma. Ann Oncol 25: 316-322, 2014.

19. Muggia FM: Teniposide: Overview of its therapeutic potential in adult cancers. Cancer Chemother Pharmacol 34 (Suppl): S127-S133, 1994.

20. Fan N, Chen BB, Zhu C, Xu XP, Lin ZG, Ma Y and Zhang J: Comparison of the efficacy of MTX-alone and combined with other drugs in treating CNS DLBCL. Fudan Univ J Med Sci 41: 81-87, 2014

21. Swerdlow SH, Campo E, Harris NL, Jaffe ES, Pileri SA, Stein H, Thiele J and Vardiman JW: WHO Classification of tumors of Hematopoietic and Lymphoid Tissues. IARC, Lyon, p439, 2008.

22. Oken MM, Creech RH, Tormey DC, Horton J, Davis TE, McFadden ET and Carbone PP: Toxicity and response criteria of the Eastern Cooperative Oncology Group. Am J Clin Oncol 5: 649-655, 1982.

23. Abrey LE, Batchelor TT, Ferreri AJ, Gospodarowicz M, Pulczynski EJ, Zucca E, Smith JR, Korfel A, Soussain C, DeAngelis LM, et al: Report of an international workshop to standardize baseline evaluation and response criteria for primary CNS lymphoma. J Clin Oncol 23: 5034-5043, 2005.

24. Jellinger KA and Paulus W: Primary central nervous system lymphomas-an update. J Cancer Res Clin Oncol 119: 7-27, 1992.

25. Reni M, Ferreri AJ, Garancini MP and Villa E: Therapeutic management of primary central nervous system lymphoma in immunocompetent patients: Results of a critical review of the literature. Ann Oncol 8: 227-234, 1997.

26. Michalski JM, Garcia DM, Kase E, Grigsby PW and Simpson JR: Primary central nervous system lymphoma: Analysis of prognostic variables and patterns of treatment failure. Radiology 176 : 855-860, 1990 .

27. Gerstner ER and Batchelor TT: Primary central nervous system lymphoma. Arch Neurol 67: 291-297, 2010. 
28. Low S and Batchelor TT: Primary central nervous system lymphoma. Semin Neurol 38: 86-94, 2018.

29. Haldorsen IS, Krossnes BK, Aarseth JH, Scheie D, Johannesen TB, Mella $\mathrm{O}$ and Espeland A: Increasing incidence and continued dismal outcome of primary central nervous system lymphoma in Norway 1989-2003: Time trends in a 15-year national survey. Cancer 110: 1803-1814, 2007

30. Makino K, Nakamura H, Kino T, Takeshima H and Kuratsu J: Rising incidence of primary central nervous system lymphoma in Kumamoto, Japan. Surg Neurol 66: 503-506, 2006.

31. Eloranta S, Branvall E, Celsing F, Papworth K, Ljungqvist M, Enblad $G$ and Ekström-Smedby $K$ : Increasing incidence of primary central nervous system lymphoma but no improvement in survival in Sweden 2000-2013. Eur J Haematol 100: 61-68, 2018.

32. Bayraktar S, Bayraktar UD, Ramos JC, Stefanovic A and Lossos IS: Primary CNS lymphoma in HIV positive and negative patients: Comparison of clinical characteristics, outcome and prognostic factors. J Neurooncol 101: 257-265, 2011.

33. Ferreri AJ, Blay JY, Reni M, Pasini F, Spina M, Ambrosetti A, Calderoni A, Rossi A, Vavassori V, Conconi A, et al: Prognostic scoring system for primary CNS lymphomas: The International Extranodal Lymphoma Study Group experience. J Clin Oncol 21: 266-272, 2003

34. Bataille B, Delwail V, Menet E, Vandermarcq P, Ingrand P, Wager M, Guy G and Lapierre F: Primary intracerebral malignant lymphoma: Report of 248 cases. J Neurosurg 92: 261-266, 2000.

35. Gelabert-González M, Castro Bouzas D, Serramito-García R, Frieiro Dantas C and Aran Echabe E: Primary central nervous system lymphoma. Neurologia 28: 283-293, 2013 (In English, Spanish).

36. Deckert M, Engert A, Brück W, Ferreri AJ, Finke J, Illerhaus G, Klapper W, Korfel A, Küppers R, Maarouf M, et al: Modern concepts in the biology, diagnosis, differential diagnosis and treatment of primary central nervous system lymphoma. Leukemia 25: 1797-1807, 2011.

37. Thiel E, Korfel A, Martus P, Kanz L, Griesinger F, Rauch M, Röth A, Hertenstein B, von Toll T, Hundsberger T, et al: High-dose methotrexate with or without whole brain radiotherapy for primary CNS lymphoma (G-PCNSL-SG-1): A phase 3 , randomised, non-inferiority trial. Lancet Oncol 11: 1036-1047, 2010.

38. O'Brien P, Roos D, Pratt G, Liew K, Barton M, Poulsen M, Olver I and Trotter G: Phase II multicenter study of brief single-agent methotrexate followed by irradiation in primary CNS lymphoma. J Clin Oncol 18: 519-526, 2000.

39. Nabors LB, Portnow J, Ammirati M, Baehring J, Brem H, Brown P, Butowski N, Chamberlain MC, Fenstermaker RA, Friedman A, et al: Central nervous system cancers, version 1.2015 . J Natl Compr Canc Netw 13: 1191-1202, 2015.

40. Fraser E, Gruenberg K and Rubenstein JL: New approaches in primary central nervous system lymphoma. Chin Clin Oncol 4: $11,2015$.

41. Kim JE, Yoon DH, Kim S, Lee DH, Kim JH, Yoon YH, Chi HS, Lee SW, Park CS, Huh J and Suh C: Relapse pattern and prognostic factors for patients with primary central nervous system lymphoma. Korean J Hematol 47: 60-66, 2012.
42. Prica A, Chan K and Cheung M: Combined modality therapy versus chemotherapy alone as an induction regimen for primary central nervous system lymphoma: A cost-effectiveness analysis. Neuro Oncol 16: 1384-1391, 2014

43. Correa DD, Maron L, Harder H, Klein M, Armstrong CL, Calabrese P, Bromberg JE, Abrey LE, Batchelor TT and Schiff D: Cognitive functions in primary central nervous system lymphoma: Literature review and assessment guidelines. Ann Oncol 18: 1145-1151, 2007.

44. Batchelor TT: Primary central nervous system lymphoma. Hematol Am Soc Hematol Educ Program 2016: 379-385, 2016.

45. Muirhead R, Murray EC, Bell SL, Stewart W and James A: Is there a role for radiotherapy in the primary management of primary central nervous system lymphoma? A single-centre case series. Clin Oncol (R Coll Radiol) 25: 400-405, 2013.

46. Swinnen LJ, O'Neill A,Imus PH, Gujar S, Schiff D, Kleinberg LR, Advani RH, Dunbar EM, Moore D and Grossman SA: Phase II study of rituximab given in conjunction with standard chemotherapy in primary central nervous system lymphoma (PCNSL): A trial of the ECOG-ACRIN cancer research group (E1F05). Oncotarget 9: 766-773, 2018.

47. Hu Y, Feng FY, Shi YK, Zhou LQ, Gu DZ and Wang QL: Primary central nervous system lymphoma: A report of 28 patients. Zhonghua Zhong Liu Za Zhi 26: 375-378, 2004 (In Chinese).

48. Zhang ET, Inman CB and Weller RO: Interrelationships of the pia mater and the perivascular (Virchow-Robin) spaces in the human cerebrum. J Anat 170: 111-123, 1990.

49. Abrey LE, Ben-Porat L, Panageas KS, Yahalom J, Berkey B, Curran W, Schultz C, Leibel S, Nelson D, Mehta M and DeAngelis LM: Primary central nervous system lymphoma: The Memorial Sloan-Kettering Cancer Center prognostic model. J Clin Oncol 24: 5711-5715, 2006.

50. Patrick LB and Mohile NA: Advances in primary central nervous system lymphoma. Curr Oncol Rep 17: 60, 2015.

51. DeAngelis LM, Yahalom J, Heinemann MH, Cirrincione C, Thaler HT and Krol G: Primary CNS lymphoma: Combined treatment with chemotherapy and radiotherapy. Neurology 40: 80-86, 1990.

52. Bierman PJ: Surgery for primary central nervous system lymphoma: Is it time for reevaluation? Oncology (Williston Park) 28: 632-637, 2014

53. Weller M, Martus P, Roth P, Thiel E and Korfel A; German PCNSL Study Group: Surgery for primary CNS lymphoma? Challenging a paradigm. Neuro Oncol 14: 1481-1484, 2012.

54. Brastianos PK and Batchelor TT: Primary central nervous system lymphoma: Overview of current treatment strategies. Hematol Oncol Clin North Am 26: 897-916, 2012.

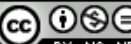

This work is licensed under a Creative Commons Attribution-NonCommercial-NoDerivatives 4.0 International (CC BY-NC-ND 4.0) License. 